\title{
22 Softwareentwicklungen zur Lernprozessbegleitung
}

\section{Oliver Samoila}

optes ist über seine beiden Förderphasen hinweg ein vielseitiges und vielschichtiges Projekt. Es wurden viele inhaltliche und methodische Angebote entwickelt, so ist optes in vielerlei Aspekten ein Forschungs- und Organisationsentwicklungs-, aber vor allem auch ein Praxisprojekt. Der projektinterne Anspruch, entwickelte Angebote inhaltlich und methodisch innovativ, zielgruppenorientiert, attraktiv in der Bedienung und verständlich in der Nutzung zu gestalten, macht optes aber auch zu einem Softwareentwicklungsprojekt.

Auch für Aspekte der Lernprozessbegleitung, der Nutzerführung und für erleichterte Kommunikation wurden diverse Softwareentwicklungen angestoßen. Dabei kann und soll hier nur eine Auswahl beleuchtet werden. ${ }^{1}$

\section{Bedarfe der Lernprozessbegleitung}

Blended-Learning- und Distance-Learning-Szenarien erfordern Maßnahmen bzw. offenbaren Bedarfe, die in klassischen Präsenzsituationen nicht oder nicht im gleichen $\mathrm{Ma} ß$ erforderlich sind. Nun gibt es eine Vielzahl von Gelingensbedingungen, die auf wissenschaftlichen (Praxis-)Untersuchungen, auf mediendidaktischen Grundannahmen oder lerntheoretischen Konstrukten fußen - der Verbund, der sich in optes ganz praktischen Problemen gegenübersah und sich aufgrund ähnlicher Problemhaushalte bildete, brachte aber auch ganz eigene gemeinsame Erfahrungswerte und Weiterentwicklungsbedarfe ein. Allen gemein war der Wunsch danach, ILIAS als ein mächtiges Lernmanagementsystem zu verwenden, mit dem diese Bedarfe bereits in Teilen zu decken waren.

\section{Mentorielle und systemseitige Unterstützung}

Lernprozessbegleitung als Akt der Beratung oder der Unterstützung wird in optesKonzepten und in der Praxis von E-Mentor*innen ${ }^{2}$ gelebt. Mentor*innen als Begleitende können vielfältige Angebote machen, schließlich gibt es in der eigenen und auch an anderen Institutionen Erfahrungswerte, womit und wodurch den Stu-

1 Generelles zum Thema Softwareentwicklung in optes in Kapitel 27.

2 Als E-Mentor*innen sind an der TH OWL Studierende höherer Semester tätig. Andere Projektpartner haben dieses Konzept auf die eigenen Rahmenbedingungen adaptiert. Zum Thema E-Mentoring siehe Kapitel 18.

Die Originalversion dieses Kapitels wurde revidiert. Ein Erratum ist verfügbar unter https://doi.org/10.1007/978-3-658-31279-4_32 
dienanfänger*innen im Übergang Schule - Hochschule und ihrem Lernalltag geholfen werden kann. Dennoch sind die Maßnahmen in der Regel darauf ausgerichtet, dass ein breites Angebot für die breite Masse in der Zielgruppe konzipiert wird und dieses über Qualität, Attraktivität und Relevanz für die einzelnen Studienanfänger*innen deren Zustimmung erfährt. Um dem Nebel der Bedarfe ein wenig Klarheit entgegenzusetzen, muss man bei den Betroffenen, den Studienanfänger*innen, ansetzen und diesen entlocken, wobei sie Unterstützung benötigen, oder sie zumindest darin anleiten, ihre eigenen Bedarfe zu entdecken und zu kommunizieren.

$\mathrm{Zu}$ diesem Zweck wurde in ILIAS beispielsweise die Möglichkeit geschaffen, über Freitexteingaben die Felder „Suche Hilfe“ und „Biete Hilfe“ auszufüllen und in den persönlichen Daten freizugeben. Diese Informationen wiederum sind durchsuchbar, um ein Matching zwischen Angeboten und Bedarfen zwischen Peers oder Studienanfänger*innen und E-Mentor*innen herzustellen. Eine Verknüpfung mit den in ILIAS hinterlegten Kompetenzbereichen (in optes den mathematischen Fähigkeiten) bietet Kriterien für die konkrete Suche.

Auch wurden Maßnahmen zur besseren Kommunikation von Selbsteinschätzungen vorgenommen. So werden Informationen über die eigenen Fähigkeiten (ob nun über Selbsteinschätzungen oder über systemseitige Datenerhebungen) über Portfolios und andere Werkzeuge ersichtlich und mit Personen, die den Lernprozess begleiten, teilbar.

Ein Instrument, welches in der zweiten Förderphase entwickelt wurde, ist der sogenannte Lernverlauf. Es handelt sich hierbei aber nicht um ein Tracking von Nutzerinteraktionen - welches, gleich ob in Lernmanagementsystemen oder in

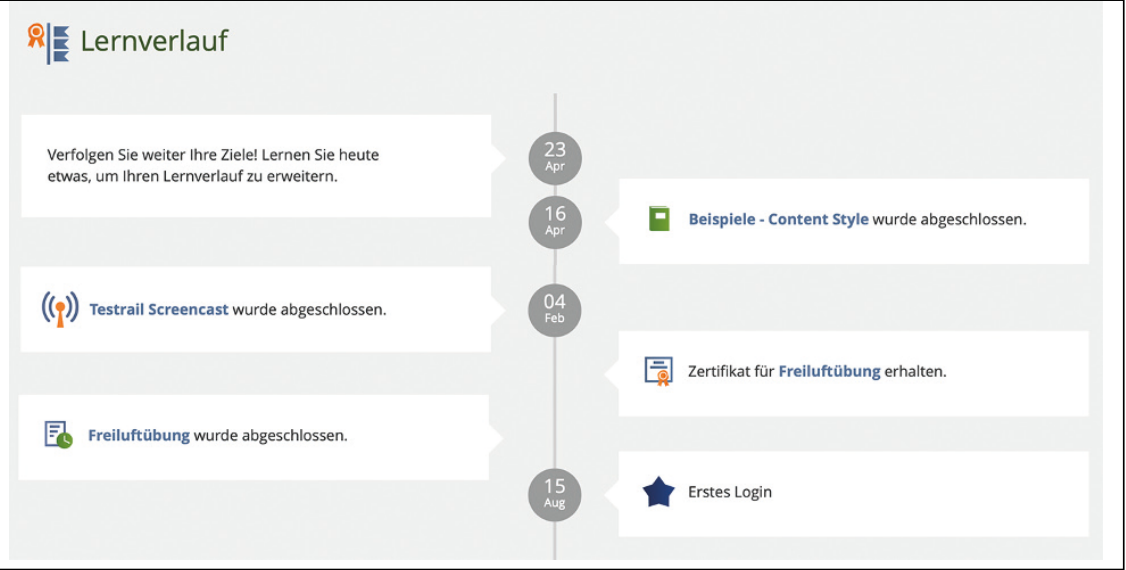

Abbildung 1: Lernerfolge im Lernverlauf 
anderen Anwendungen stattfindet, kein einfacher Vorgang ist und sich zu Recht einer Vielzahl von datenschutzrelevanten Hürden gegenübersieht. Ziel war es, die Daten der Lernenden, die bereits in ILIAS vorliegen, so zu arrangieren, dass Lernende sie übersichtlich, verständlich und erfassbar vorliegen haben - und den Vorgang des Teilens dieser Information selbst kontrollieren können. ILIAS bietet als Kategorien von Lernerfolgen Badges, Kompetenzerhebungen, Lernfortschritt und Lernziele sowie Zertifikate. Alle Lernerfolge zusammen werden dem Lernenden chronologisch und direkt zugänglich im eigenen individuellen Lernverlauf angeboten.

\section{Nutzerführung und -leitung}

Neben der Zielsetzung, Lernenden in der Selbstwahrnehmung und -reflexion Anleitung zu geben, ist ein weiterer Handlungsstrang die bessere Nutzerführung im Lernmanagementsystem. Beispielhaft werden dafür die Weiterentwicklungen um das persönliche Profil und die Übung in ILIAS vorgestellt.

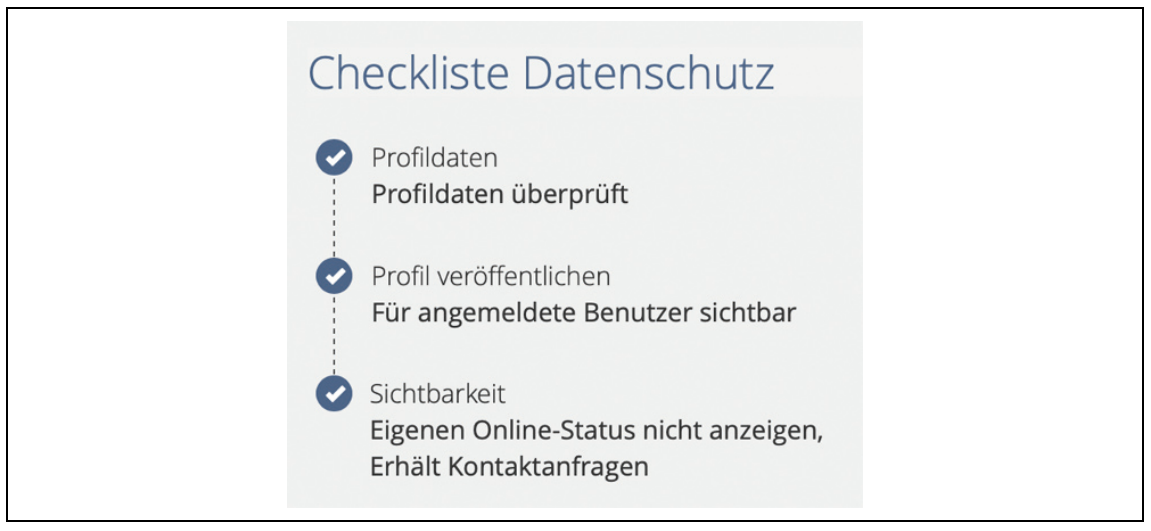

Abbildung 2: Workflow für das persönliche Profil

Anonymität und persönliche (möglichst individuelle) Lernprozessbegleitung sind zwei nur schwer miteinander zu vereinbarende Ziele. In den Projektjahren hat sich immer wieder gezeigt, dass ein persönlicher Kontakt zu den Lernbegleitenden „Tim“ und „Lisa“ besser funktioniert als zum „Lernbegleitungs-Team“. Ebenso verhält es sich auf der Seite der Lernenden auch untereinander. Sich in digitalen Räumen zu bewegen und Kommiliton*innen oder Lernbegleitende zu erkennen, ansprechen zu können und mit ihnen zusammenarbeiten zu können, funktioniert leichtfüßiger und ist authentischer, als sich einer anonymen Masse gegenüber zu sehen. 
Unsere heutigen Studierenden sind in der Regel firm darin, mit ihren persönlichen Daten und deren Veröffentlichung in sozialen oder beruflichen Netzwerken umzugehen. Worin wir sie allerdings unterstützen mussten, ist, ihre Daten auch in einem System wie ILIAS zu Lernzwecken zu veröffentlichen. In einem zweistufigen Ausbauprozess wurden zunächst Hinweise zum persönlichen Profil ermöglicht, die administrationsseitig eine Erläuterung zu Sinn und Zweck sowie Anleitungen zum Umgang mit den Daten enthalten können, und daraufhin ein Workflow etabliert, der durch die Eingabe von persönlichen Daten und deren Veröffentlichung sowie durch persönliche Einstellungen, die datenschutzrelevant sind, führt. Letzteres ist ein bekanntes Pattern aus sozialen und beruflichen Netzwerkdiensten oder Online-Portalen.

Die Nutzung eines Lernmanagementsystems aus Konsumentenperspektive ist eine Sache, der Wechsel in die Perspektive der Autorenschaft aber noch einmal etwas ganz anderes. Ein bereits oben und in Kapitel 5 beschriebener Auftrag an die Lernenden ist die Kommunikation über die eigenen Bedarfe und das eigene Lernen in Form von Reflexion. Reflexives Schreiben in einem Blended-LearningSzenario ist eine hohe Anforderung an Studierende und für Studienanfänger*innen in einem Distance-Learning-Szenario umso höher. Um Hürden im Bearbeitungsund Schreibprozess, auch anhand von Leitfragen und Vorgaben, zumindest im Bereich der Nutzung des Lernmanagementsystems abzubauen, wurde eine deutlich stärkere Nutzerführung konzipiert, entwickelt und auch bereits erprobt. Als Mittel der Wahl wurden für die Begleitung des reflexiven Schreibprozesses in ILIAS Übungen identifiziert. Diese führen mit Arbeitsanweisungen parallel zum Schreibprozess durch eben diesen hindurch. Auch wenn es nur wenig spektakulär anmutet, so ist die Kombination aus Schreiben bei gleichzeitiger Verfügbarkeit von Leitfragen und Ergänzungsmaterialien (wie bspw. Texten, Vorlagen, Audio \& Video) keine selbstverständliche Arbeitsumgebung - gleich wohl auch auf mobilen Endgeräten wie Tablets verfügbar. Lernende werden so step-by-step von der Arbeitsanweisung über das Schreiben bis hin zur Einreichung oder zum Teilen mit Lernprozessbegleitenden durch den Prozess geführt. Anschließend stehen den EMentor*innen wie gewohnt verschiedene Modi des Feedbacks zur Verfügung.

\section{Kommunikationsmittel in der Lernprozessbegleitung}

Der Umgang mit E-Mails ist alltäglich, auch Foren sind für heutige Lernende keine Besonderheit mehr - nur handelt es sich bei beiden Formaten um asynchrone Kommunikationskanäle. Bereits Ende der ersten Förderphase wurde daher viel Aufwand in die Renovierung des bereits in ILIAS bestehenden Chats investiert. Lernende sind aus ihrem Alltag die Verwendung von Messenger-Diensten und Chats in sozialen Netzwerken gewohnt und darin geübt - nur handelt es sich dabei nie um einen in die Lernumgebung integrierten Vorgang. 


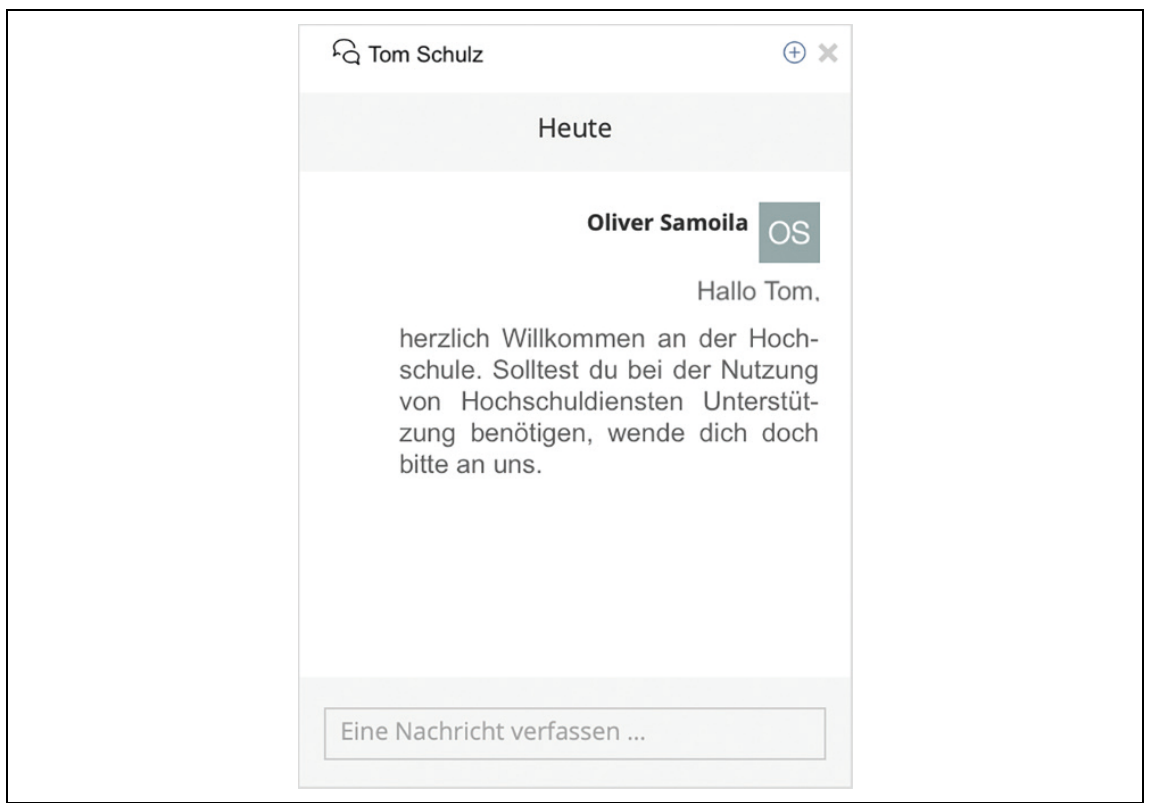

Abbildung 3: On-Screen-Chat in ILIAS

Der Vorgang direkter vor allem synchroner Kommunikation mit Lernprozessbegleitenden (aber auch unter Kommiliton*innen) im Lernmanagementsystem selbst dient nicht nur der Akzeptanz des Systems, sondern lässt Kontakte mit Ansprechpersonen im digitalen Raum zu, die sonst gegebenenfalls gar nicht entstünden. Lernenden steht ein niedrigschwelliger und einfach zu bedienender Chat zur Verfügung, der Zugriff auf Kontakte ermöglicht, die auch gerade online sind. Zusätzlich lassen sich auch Chatsitzungen mit mehreren Personen durchführen. 
Open Access Dieses Kapitel wird unter der Creative Commons Namensnennung 4.0 International Lizenz (http://creativecommons.org/licenses/by/4.0/deed. de) veröffentlicht, welche die Nutzung, Vervielfältigung, Bearbeitung, Verbreitung und Wiedergabe in jeglichem Medium und Format erlaubt, sofern Sie den/ die ursprünglichen Autor(en) und die Quelle ordnungsgemäß nennen, einen Link zur Creative Commons Lizenz beifügen und angeben, ob Änderungen vorgenommen wurden.

Die in diesem Kapitel enthaltenen Bilder und sonstiges Drittmaterial unterliegen ebenfalls der genannten Creative Commons Lizenz, sofern sich aus der Abbildungslegende nichts anderes ergibt. Sofern das betreffende Material nicht unter der genannten Creative Commons Lizenz steht und die betreffende Handlung nicht nach gesetzlichen Vorschriften erlaubt ist, ist für die oben aufgeführten Weiterverwendungen des Materials die Einwilligung des jeweiligen Rechteinhabers einzuholen.

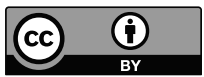

